OPEN

SUBJECT AREAS:

OPTICAL TECHNIQUES

NANOWIRES

SUB-WAVELENGTH OPTICS

DESIGN, SYNTHESIS AND

PROCESSING

Received

9 August 2013

Accepted

13 January 2014

Published

28 January 2014

Correspondence and requests for materials should be addressed to X.X. (xxu@purdue.edu)

\title{
Sub-diffraction Laser Synthesis of Silicon Nanowires
}

\author{
James I. Mitchell, Nan Zhou, Woongsik Nam, Luis M. Traverso \& Xianfan Xu
}

School of Mechanical Engineering, Birck Nanotechnology Center Purdue University, West Lafayette, Indiana 47907.

We demonstrate synthesis of silicon nanowires of tens of nanometers via laser induced chemical vapor deposition. These nanowires with diameters as small as $60 \mathrm{~nm}$ are produced by the interference between incident laser radiation and surface scattered radiation within a diffraction limited spot, which causes spatially confined, periodic heating needed for high resolution chemical vapor deposition. By controlling the intensity and polarization direction of the incident radiation, multiple parallel nanowires can be simultaneously synthesized. The nanowires are produced on a dielectric substrate with controlled diameter, length, orientation, and the possibility of in-situ doping, and therefore are ready for device fabrication. Our method offers rapid one-step fabrication of nano-materials and devices unobtainable with previous CVD methods.

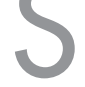

ince the initial description of nanowire synthesis via the vapor-liquid-solid (VLS) mechanism ${ }^{1,2}$, nanowires have garnered significant attention as a means of constructing nano-devices with a wide breadth of application $s^{3-5}$ due to their fine dimensions ${ }^{6}$ and variety of materials and structures ${ }^{7}$. Despite this potential, the lack of control in growth, placement and orientation compounded by potential contamination from metal catalysts still hinders more widespread implementation. Guiding nanowire formation ${ }^{8-10}$ and eliminating metal precursors ${ }^{11}$ have been attempted, but have not entirely overcome these obstacles. Here, we demonstrate synthesis of silicon nanowires with diameters of $60 \mathrm{~nm}$ via laser induced chemical vapor deposition (CVD). We produce nanowires by utilizing interference between incident laser radiation and surface scattered radiation that generates spatially confined, periodic heating needed for obtaining nanowires with diameters far below the diffraction limit. By controlling the intensity and polarization direction of the incident laser radiation, single or multiple parallel nanowires are synthesized on a dielectric substrate with controlled diameter, length, orientation, and the possibility of in-situ doping, making them ready for device fabrication.

Direct chemical synthesis of materials by laser illumination has been used to fabricate valuable electrical and photonic components ${ }^{12-14}$, but the feature sizes were limited to hundreds of nanometers due to the diffraction limit of light. On the other hand, laser based methods including multiphoton polymerization ${ }^{15}$, stimulated emission depletion of polymers ${ }^{16,17}$, and near field plasmonic processing ${ }^{18-20}$ have achieved features with critical dimensions of tens of nanometers, but these methods generally cannot be directly implemented for nanomaterials growth. It is therefore useful to develop a method capable of combining the fine features of sub diffraction limit laser growth with direct material synthesis. Here we describe a laser-based method to produce silicon nanowires with widths of about $60 \mathrm{~nm}$, directly on a dielectric substrate, with controlled length, orientation, and in-situ doping.

\section{Results}

The key to producing these sub-diffraction limited nanowires was to utilize the interference effect between the incident laser beam and the surface scattered laser radiation. In an environment of silane gas flow, high numerical aperture Fresnel phase zone plates provided a near diffraction-limited focal spot, and due to the aforementioned interference effect, spatially periodic heating occurred within the well-defined laser spot. The elevated temperature decomposed the silane gas (Fig. 1a-b), and translating the substrate defined the length and orientation of the nanowire. Figures $1 \mathrm{c}-\mathrm{d}$ illustrate the radiation scattering by the initially formed nanowire, which caused the interference pattern. By appropriately controlling the laser power, single, double, or triple nanowires can be produced in one focal spot (Figs. 2a-c). Particularly, a single nanowire forms by allowing only the substrate surface corresponding to the central high intensity area to exceed the silane decomposition temperature. These individual nanowires have a semicircular cross-section with a height of around 30 nanometers (Fig. 2g). The high numerical aperture of the zone plates was critical to forming a single nanowire because the intensity peak of the 
a

Laser heats substrates surface

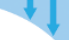
,

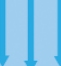

Resulting interference dictates nanowire shape

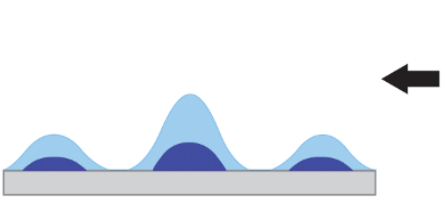

C

Radiation scatteres off nanowire

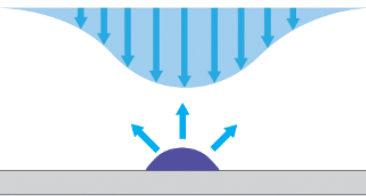

Figure 1 Schematic of radiation interference for silicon nanowire diameter control. (a), Laser irradiation incident on the substrate surface induces a heat increase. (b), The increase in heating causes initiation of nanowire synthesis. (c), As the initial nanowire begins to develop radiation is scattered off the nanowire surface. (d), The incident beam and the scattered radiation interfere and the resulting intensity distribution confines the area where the nanowire forms.

focal spot must be narrow enough so that only the central peak of the interference fringes has sufficient intensity for heating the substrate in excess of the silane deposition temperature.

To further illustrate the forming of the nanowire patterns, Figs. $2 \mathrm{~d}-\mathrm{f}$ show the nanowire shape and orientation dependence on the polarization of the incident radiation. Fig. $2 \mathrm{~d}$ and Fig. $2 \mathrm{e}$ demonstrate nanowire patterns 45 degrees from the vertical direction and in the vertical direction, respectively, which match the laser polarization direction. We also tested the effect of using circularly polarized light, resulting in nanowires hundreds of nanometers in diameter. With in situ doping of boron using diborane together with silane, these thick wires which were essentially agglomerations of thinner nanowires, yielded rough surfaces (Fig. 2f) which gave improved sensitivity for chemical detection ${ }^{21}$.
The laser induced nano-structures used here for nanowire formation, have been observed elsewhere for high power situations where intense laser pulses ablate material to form nanoscale ripples or grooves on the substrate. In these other cases, the substrate material largely determines both the periodicity $(\Lambda)$ of the ripples as well as the direction they form relative to the polarization direction of the incident laser radiation ${ }^{22}$. The periodicity is often divided into two regimes depending on the wavelength $(\lambda)$ : low spatial frequency for $\lambda>\Lambda>0.4 \lambda$ and high spatial frequency for $0.4 \lambda>\Lambda^{23}$. Low spatial frequency ripples are often described as forming when the incoming laser radiation interferes with radiation scattered from the substrate to create high radiation intensity distributions that remove the material ${ }^{24}$. It has been shown that this restructuring can act as a feedback mechanism which decreases the ripple spacing as the number of laser pulses increase ${ }^{22,23,25}$. On some dielectric surfaces, and in particular silicon dioxide, low spatial frequency ripples form parallel to the laser's electric field polarization direction ${ }^{22}$. Interference between incident radiation and surface or volume plasmons caused by the incident radiation has been suggested as part of the mechanism for producing ripples on metal surfaces ${ }^{26}$, on semiconductor surfaces $^{23,25}$, or for dielectric materials where the laser power is high enough to generate sufficient free electrons to cause the material to act metallically ${ }^{23,27}$. In these cases, the ripples are perpendicular to the polarization direction of the incident laser beam since interference occurs when the component of the electrical field of the surface plasmon, along its propagation direction and perpendicular to the ripples, is in the same direction as the electrical field of the incoming laser.

To explain the nanowire formation process in our case where the laser fluences are far below the ablation threshold, we carried out numerical computations using the frequency-domain finite-element method (FEM). The laser fluences used for these calculations ranged from 0.012 to $0.029 \mathrm{~J} / \mathrm{cm}^{2}$, corresponding to the range of laser fluences used for the formation of single and triple nanowires respectively (Supplementary Section S4) and we estimate the temperature increase to be in line with what would be expected for silane decomposition (Supplementary Section S5). Figure 3a shows the calculated $\left|E_{x}\right|^{2}$ distribution along the dashed line in the inset, and illustrates the interference effect causing a center peak and two side lobes about $180 \mathrm{~nm}$ away from the center. The width of the center high intensity a

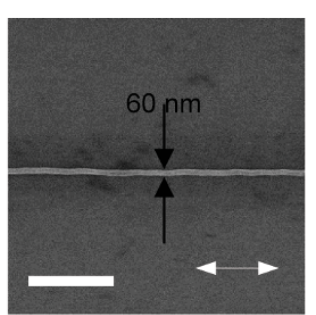

d

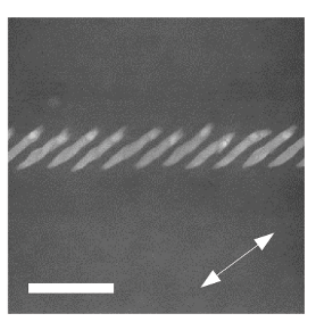

b

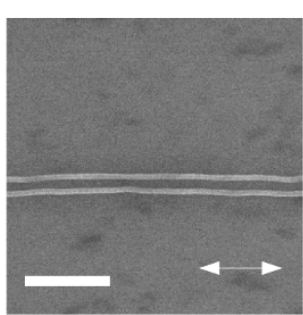

e

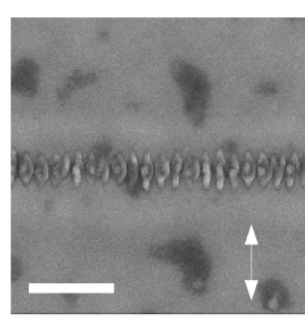

C

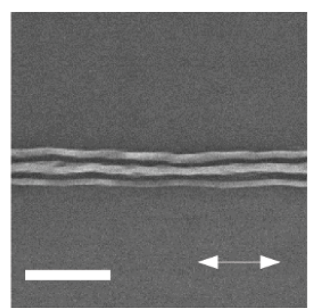

f

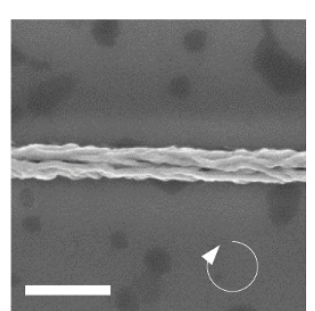

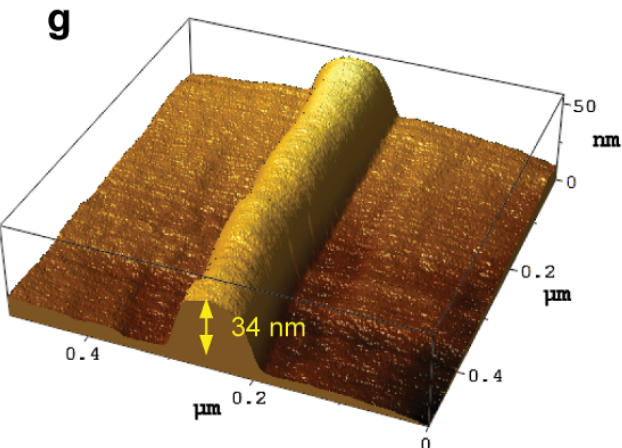

Figure $2 \mid$ Polarization effect on nanowire. White arrows indicate the electric field polarization direction. SEM images of nanowires grown from horizontally polarized laser radiation show: (a), Single (b), double and (c), triple nanowires, and the single wire has a width of 60 nanometers. SEM images demonstrate the polarization dependence of nanowire patterns for (d), linear polarization 45 degrees from the scanning direction, at (e), linear polarization 90 degrees from the scanning direction, and for (f), circular polarization. (g), Atomic force microscope image of a single written nanowire demonstrates the height at $34 \mathrm{~nm}$. All scale bars are $1 \mu \mathrm{m}$. 

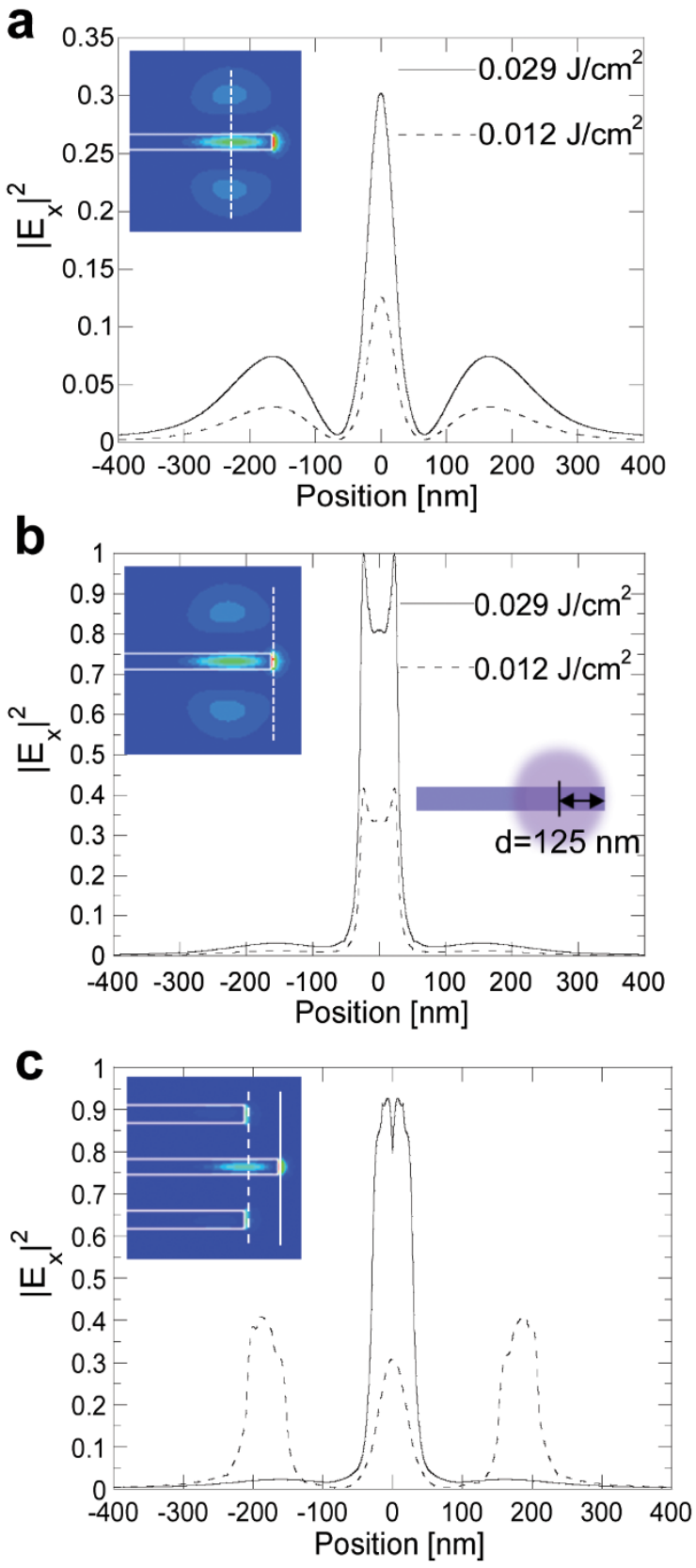

Figure $3 \mid$ Computational results of electrical field $\left(\left|E_{x}\right|^{2}\right)$ distributions during nanowire synthesis. The distribution for a single nanowire at two laser fluences is displayed along two scan lines in (a) and (b). The left inset in (b) shows the highest intensity is located at the wire tip. (c), Intensity distribution for triple nanowires. In (c) the spacing between wires is $180 \mathrm{~nm}$ and the central wire is $125 \mathrm{~nm}$ longer than those wires to the side. The simulated wires are $60 \mathrm{~nm}$ wide, $30 \mathrm{~nm}$ high with a semi-circular cross section. The intensity was normalized to the maximum value at the fluence of $0.029 \mathrm{~J} / \mathrm{cm}^{2}$. For all simulations, the electric field polarization of the incoming laser was parallel to the wires.

region is restricted by the interference effect to about $59 \mathrm{~nm}$ full width half maximum. If the laser fluence increases, the intensity at the two side lobes will exceed the threshold to form triple lines. The 2$\mathrm{D}\left|\mathrm{E}_{\mathrm{x}}\right|^{2}$ distribution on the silicon dioxide surface (inset of Fig. 3a) shows high intensities at the wire tip and the two side lobe-like areas. The field at the tip of the wire has higher intensity, shown in the $\left|E_{x}\right|^{2}$ distribution along the dashed line in the inset of Fig. 3b, and this high intensity at the tip leads the nanowire formation. Similarly, for the formation of three wires, the electrical field distribution in Fig. $3 \mathrm{c}$ shows the high intensity areas at the tips of the three wires causing the nanowire growth.

The explanation given above is further supported by noting that at the beginning of nanowire growth, there is no scattering and no interference, therefore creating a large initial wire diameter (Fig. 4a). Although the initial diameter is large, it is quickly reduced as the interference begins to affect formation. Correspondingly, the wire termination should demonstrate no enlargement of the diameter as verified in Fig. 4b. Simulations also show that for triple wire formations the center wire is longer than the two side wires (Fig. 3a inset). The highest intensities of the two side lobes are at the same lengthwise locations as the center of the laser spot, whereas the highest intensity along the center nanowire is in front of the laser spot, and this effect is seen at the end of the triple nanowires when the laser radiation is terminated (Fig. 4c). If the polarization direction of the incident laser beam is not completely parallel to the substrate scanning direction, the number of nanowires can fluctuate as the front of the nanowire moves off the center of the laser irradiation, and can be responsible for the formation of two nanowires where each nanowire provides the scattered radiation for the other (Fig. S1).

We emphasize that for our nanowire synthesis the interference between the incident and scattered laser radiation forms interference fringes for nanowire synthesis, and the plasmonic effect does not play a role. We carried out non-linear ultrafast pulse propagation calculations in silicon dioxide (Supplementary Section S2) considering the multi-photon absorption effect, the resulting free electron density, and the modifications to the dielectric constant. At the laser fluences for nanowire synthesis, the free electron density is less than $6 \times$ $10^{19} \mathrm{~cm}^{-3}$, which is more than two orders of magnitude lower than the critical free electron density for the material to behave metallically. Therefore, surface plasmons do not contribute to the interference effect we demonstrate here, in contrast to the case of femtosecond laser ablation of most dielectric materials ${ }^{23,27}$. Furthermore, the fringes formed by the interference between the incident radiation and the induced surface plasmons are perpendicular to the laser polarization direction, whereas the nanowires we grew form parallel to the polarization direction. We verify using numerical simulation that if the laser power is high enough, interference fringes can form perpendicular to the polarization direction (Fig. S2).

\section{Discussion}

While other processes for synthesizing nanowires are limited in placement precision, resolution, or flexibility due to the stringent requirements to create features with critical dimensions of tens of nanometers, our laser synthesis method for producing nanowires provides a means for creating nanowires laying horizontally on a dielectric substrate which electrically insulates the nanowires, with precise location, length, orientation, and in-situ doping allowing for easy integration of these nanowires into devices. With typical laser powers on the order of watts, an array of Fresnel's zone plates can generate hundreds of light spots for parallel writing to scale up the nanowire synthesis process. This could be of particular use in producing devices on transparent insulating materials or for thin film transistors where precisely placed nanowires could simplify manufacturing and increase device performance ${ }^{28}$.

In this work we demonstrated a laser induced CVD method capable of fabricating nanowires far below the diffraction limit with widths of only $60 \mathrm{~nm}$ using far-field optics. We utilized the interference between scattered laser radiation and radiation incident on the substrate surface to obtain these narrow widths. We confirmed the nanowire formation mechanism by performing electromagnetic field simulations which agreed with the phenomena observed in the experiments. This nanowire fabrication method provides a means to precisely place nanowires for a device and can serve as a useful platform for nanofabrication of a variety of materials and devices. 

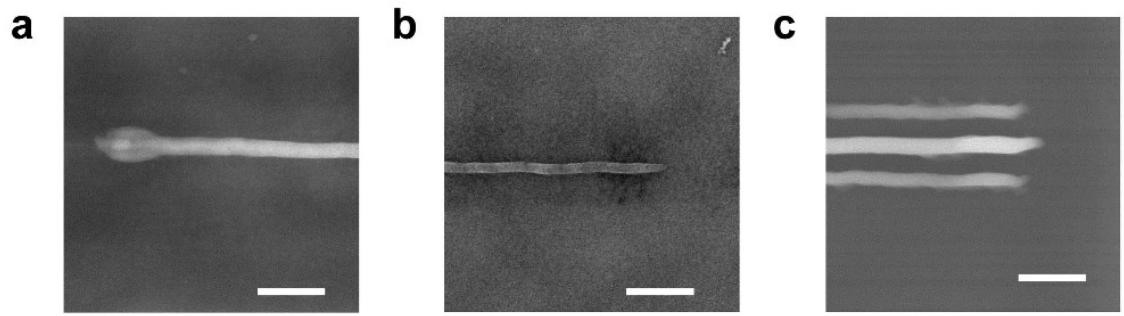

Figure $4 \mid$ Nanowire formation process. (a), Synthesis at the start of the nanowire produces a slightly larger diameter until the scattered light from the nanowire is sufficient to generate stable interference. (b), SEM image showing the nanowire termination demonstrates that the larger diameter is only at the beginning of the nanowire and not at the end. (c), At the termination of a triple nanowire the side nanowires are offset from the center nanowire. All scale bars are $400 \mathrm{~nm}$.

\section{Methods}

We used a Ti:sapphire femtosecond laser that was frequency doubled using a BBO crystal to a wavelength of $395 \mathrm{~nm}$ with a repetition rate of $80 \mathrm{MHz}$ and pulse duration measured before frequency doubling using an autocorrelator of $38 \mathrm{fs}$. We used a Fresnel's zone plate with a focal length of $50 \mu \mathrm{m}$ and numerical aperture of 0.95 to focus the laser and we used a camera and optical microscope to measure a diffraction limited spot size with a full width half maximum of $251 \mathrm{~nm}$. Silane gas provided the silicon source from a 10 percent silane in hydrogen mixture. Diborane was also used for making the nanowires electrically conductive and the diborane we used had a concentration of $100 \mathrm{ppm}$ balanced in hydrogen. The combined flow rate of silane and dibornae was $6 \mathrm{sccm}$, and the pressure during nanowire growth was 30 Torr. SEM imaging was done using a Hitachi S-4800 FESEM and nanowire widths were determined using SEM images in combination with an imaging software tool.

The substrates used for nanowire growth were $1 \mathrm{~mm}$ thick quartz coated with $350 \mathrm{~nm}$ of low pressure CVD grown amorphous silicon at $545^{\circ} \mathrm{C}$ for 260 minutes. This amorphous silicon was oxidized at $1100^{\circ} \mathrm{C}$ for 130 minutes to yield $200 \mathrm{~nm}$ of thermally grown silicon dioxide on $200 \mathrm{~nm}$ of polysilicon. Laser absorption occurred in both the top silicon dioxide layer via two-photon absorption and in the underlying polysilicon layer. Heat from the polysilicon raised the temperature in the area of the laser spot, but not high enough to decompose silane, and the heat from the radiation absorbed in the silicon dioxide layer then provided a sufficient temperature increase only in the areas of high interference fringe intensity. Without the polysilicon layer, the laser intensity needed for nanowire synthesis was much higher, and with the polysilicon only a few milliwatts were needed to produce nanowires.

Zone plates for laser focusing were made using a process similar to that described by Gil et al $^{29}$ where we spin coated hydrogen silsesquioxane (HSQ) on a $1 \mathrm{~mm}$ thick quartz substrate with an indium tin oxide (ITO) coating. We used hexamethyldisilazane (HMDS) to promote adhesion between HSQ and the ITO layer. The HSQ was patterned with electron beam lithography and baked to solidify the developed HSQ. The final zone plate thickness was $425 \mathrm{~nm}$.

1. Wagner, R. S. \& Ellis, W. C. Vapor-liquid-solid mechanism of single crystal growth. Appl. Phys. Lett. 4, 89 (1964).

2. Morales, A. M. \& Lieber, C. M. A laser ablation method for the synthesis of crystalline semiconductor nanowires. Science 279, 208-211 (1998).

3. Cui, Y., Wei, Q., Park, H. \& Leiber, C. M. Nanowire nanosensors for highly sensitive and selective detection of biological and chemical species. Science 293, 1289-92 (2001).

4. Yan, R., Gargas, D. \& Yang, P. Nanowire photonics. Nat. Photonics 3, 569-576 (2009).

5. Tian, B. et al. Coaxial silicon nanowires as solar cells and nanoelectronic power sources. Nature 449, 885-889 (2007).

6. Ma, D. D. D., Lee, C. S., Au, F. C. K., Tong, S. Y. \& Lee, S. T. Small-diameter silicon nanowire surfaces. Science 299, 1874-7 (2003).

7. Lauhon, L. J., Gudiksen, M. S., Wang, D. \& Lieber, C. M. Epitaxial core-shell and core-multishell nanowire heterostructures. Nature 420, 57-61 (2002).

8. Ryu, S.-G. et al. On demand shape-selective integration of individual vertical germanium nanowires on a $\mathrm{Si}(111)$ substrate via laser-localized heating. $A C S$ Nano 7, 2090-8 (2013).

9. Tsivion, D., Schvartzman, M., Popovitz-Biro, R., v. Huth, P. \& Joselevich, E. Guided growth of millimeter-long horizontal nanowires with controlled orientations. Science 333, 1003-7 (2011).

10. Mårtensen, T., Borgström, M., Seifert, W., Ohlsson, B. J. \& Samuelson, L. Fabrication of individually seeded nanowire arrays by vapor-liquid-solid growth. Nanotechnology 14, 1255-1258 (2003).

11. Kim, B.-S. et al. Catalyst-free growth of single-crystal silicon and germanium nanowires. Nano Lett. 9, 864-9 (2009).

12. Bäuerle, D. Laser Processing and Chemistry (Springer-Verlag, Berlin, 1996).

13. Rill, M. S. et al. Photonic metamaterials by direct laser writing and silver chemical vapor deposition. Nat. Mater. 7, 543-6 (2008).

14. Baum, T. H. \& Comita, P. B. Laser-induced chemical vapor deposition of metals for microelectronics technology. Thin Solid Films 218, 80 (1992).

15. Cumpston, B. H. et al. Two-photon polymerization initiators for threedimensional optical data storage and microfabrication. Nature 398, 51-54 (1999).

16. Andrew, T. L., Tsai, H.-Y. \& Menon, R. Confining light to deep subwavelength dimensions to enable optical nanopatterning. Science 324, 917-21 (2009).

17. Gan, Z., Cao, Y., Evans, R. A. \& Gu, M. Three-dimensional deep sub-diffraction optical beam lithography with $9 \mathrm{~nm}$ feature size. Nat. Commun. 4, 2061 (2013).

18. Srituravanich, W. et al. Flying plasmonic lens in the near field for high-speed nanolithography. Nat. Nanotechno. 3, 733-7 (2008).

19. Wang, L., Uppuluri, S. M., Jin, E. X. \& Xu, X. Nanolithography using high transmission nanoscale bowtie apertures. Nano Lett. 6, 361-364 (2006).

20. Liao, X. et al. Desktop nanofabrication with massively multiplexed beam pen lithography. Nat. Commun. 4, 2103 (2013).

21. Nam, W., Mitchell, J. I., Tansarawiput, C., Qi, M. \& Xu, X. Laser direct writing of silicon field effect transistor sensors. Appl. Phys. Lett. 102, 093504 (2013).

22. Bönse, J., Krüger, J., Höhm, S. \& Rosenfeld, A. Femtosecond laser-induced periodic surface structures. J. Laser Appl. 24, 042006 (2012).

23. Huang, M., Zhao, F., Chong, Y., Xu, N. \& Xu, Z. Origin of laser-induced nearsubwavelength ripples: Interference between surface plasmons and incident laser. ACS Nano 3, 4062-70 (2009).

24. Sipe, J. E., Young, J. F., Preston, J. S. \& van Driel, H. M. Laser-induced periodic surface structure. I. Theory. Phys. Rev. B 27, 1141-1153 (1983).

25. Bönse, J., Rosenfeld, A. \& Krüger, J. On the role of surface plasmon polaritons in the formation of laser-induced periodic surface structures upon irradiation of silicon by femtosecond-laser pulses. J. Appl. Phs. 106, 104910 (2009).

26. Brueck, S. \& Ehrlich, D. Stimulated surface-plasma-wave scattering and growth of a periodic structure in laser-photodeposited metal films. Phys. Rev. Lett. 48, 1678-1682 (1982).

27. Shimotsuma, Y., Kazansky, P. G., Qiu, J. \& Hirao, K. Self-organized nanogratings in glass irradiated by ultrashort light pulses. Phys. Rev. Lett. 91, 247405 (2003).

28. Sun, D.-M. et al. Flexible high-performance carbon nanotube integrated circuits. Nat. Nanotechno. 6, 156-161 (2011).

29. Gil, D., Menon, R. \& Smith, H. Fabrication of high-numerical-aperture phase zone pales with a single lithography exposure and no etching. J. Vac. Sci. Technol. B 21, 2956-2960 (2003).

\section{Acknowledgments}

We acknowledge the support of the Defense Advanced Research Projects Agency (Grant No. N66001-08-1-2037) and the National Science Foundation (Grant No. CMMI-1120577).

\section{Author contributions}

X.X. conceived of laser synthesis of silicon nanowires and guided the experiments and system design. J.M. performed the experiments on nanowire synthesis and the corresponding analysis. N.Z. performed numerical simulations. W.N. fabricated the substrates and W.N. and L.T. fabricated zone plates for nanowire synthesis. J.M., N.Z., W.N. and X.X. co-wrote the paper.

\section{Additional information}

Supplementary information accompanies this paper at http://www.nature.com/ scientificreports

Competing financial interests: The authors declare no competing financial interests. How to cite this article: Mitchell, J.I., Zhou, N., Nam, W., Traverso, L.M. \& Xu, X. Sub-diffraction Laser Synthesis of Silicon Nanowires. Sci. Rep. 4, 3908; DOI:10.1038/ srep03908 (2014).

This work is licensed under a Creative Commons AttributionNonCommercial-ShareAlike 3.0 Unported license. To view a copy of this license, visit http://creativecommons.org/licenses/by-nc-sa/3.0 